\title{
Alterations in diabetes mellitus gene expression in the development and progression of Alzheimer's disease: The PI3KJAkt pathway link
}

\author{
Karla A. Hernández-Contreras, Mª Elena Hernández-Aguilar, Deissy Herrera-Covarrubias, \\ Fausto Rojas-Durán, and Gonzalo E. Aranda-Abreu* \\ Instituto de Investigaciones Cerebrales, Universidad Veracruzana, Xalapa, Veracruz, Mexico
}

\begin{abstract}
Considered a neurodegenerative disease, Alzheimer's disease (AD) involves a physiopathological process characterized by the presence of the beta-amyloid peptide, hyperphosphorylated Tau protein, and neuroinflammation. Diabetes mellitus (DM) is an endocrine disease characterized by insulin resistance, where decreased production of this hormone causes a constant state of hyperglycemia. Although it is recognized that $D M$ is a risk factor for the development of $A D$, the compression of the mechanisms involved is not completely understood. The present review evaluates the information acquired from primary and secondary sources, focusing on the alterations in gene expression associated with $A D$ as well as the alterations in gene expression associated with DM, to later highlight the influence that these types of alterations developed in patients with DM can have on both the development and progression of AD. Finally, we point out those alterations that impact the phosphatidyl Inositol 3 Kinase/Akt (PI3K/Akt) pathway, which seems to intervene in the physiopathological process of both diseases, considering that the compression of these gene alterations can help us understand the intricate link between $D M$ and $A D$.
\end{abstract}

Key words: Alzheimer's disease. Diabetes mellitus. Akt pathway.

\section{Alteraciones de la expresión génica de la diabetes mellitus en el desarrollo y progresión de la enfermedad de Alzheimer: el vínculo de la vía PI3K/Akt}

\section{Resumen}

Considerada una enfermedad neurodegenerativa, la enfermedad de Alzheimer (EA) implica un proceso fisiopatológico caracterizado por la presencia del péptido beta amiloide, la proteína Tau hiperfosforilada y la neuroinflamación. La diabetes mellitus (DM) es una enfermedad endocrina caracterizada por la resistencia a la insulina, donde la disminución de la producción de esta hormona causa un estado constante de hiperglucemia. Aunque se reconoce que la DM es un factor de riesgo para el desarrollo de la EA, la compresión de los mecanismos involucrados no se comprende completamente. En el presente trabajo se evalúa la información adquirida de fuentes primarias y secundarias, centrándose en las alteraciones de la expresión génica asociadas a la EA, así como en las alteraciones de la expresión génica asociadas a la DM, para posteriormente destacar la influencia que este tipo de alteraciones desarrolladas en pacientes con DM pueden tener

Correspondence:

*Gonzalo E. Aranda-Abreu

E-mail: garanda@uv.mx

DOI: $10.24875 / R M N .20000087$
Available online: $01-10-2021$

Rev Mex Neuroci. 2021;22(5):184-195 www.revmexneurociencia.com 1665-5044/ @ 2020 Academia Mexicana de Neurología A.C. Published by Permanyer. This is an open access article under the CC BY-NC-ND license (http://creativecommons.org/licenses/by-nc-nd/4.0/). 
tanto en el desarrollo como en la progresión de la EA. Por último, señalamos aquellas alteraciones que inciden en la vía del fosfatidilinositol-3-quinasa/Akt (PI3K/Akt), que parece intervenir en el proceso fisiopatológico de ambas enfermedades, considerando que la compresión de estas alteraciones genéticas puede ayudarnos a entender el intrincado vínculo entre la $D M$ y la $E A$.

Palabras clave: Enfermedad de Alzheimer. Diabetes mellitus. Vía de Akt.

\section{Introduction}

Alzheimer's disease (AD) and diabetes mellitus (DM) are diseases whose worldwide incidence is considerably significant, and projections estimate dramatic increases in the population affected by these diseases over the next 10 years ${ }^{1}$.

Considered a neurodegenerative disease, $A D$ is characterized by progressive memory loss accompanied by aphasia, apraxia, agnosia, and mood alterations ${ }^{2}$. From the molecular perspective, three important points can be emphasized in the physiopathological process of this disease: the $\beta$-amyloid peptide $(A \beta)$, the hyperphosphorylated Tau protein ( $\mathrm{p}$-Tau), and the neuroinflammation ${ }^{3}$.

On the other hand, DM is an endocrine disease characterized by a sustained state of hyperglycemia that gives rise to multiple clinical manifestations of which cognitive deterioration can be highlighted, which is closely related to the level of hyperglycemia and the period of exposure to this disease ${ }^{4}$. Other important alterations in the physiopathological process of DM are insulin resistance (iR) and the decrease or null production of this hormone ${ }^{5}$.

To do so, the alterations inherent to AD and DM are explained as independent entities, and subsequently those alterations in gene expression that both diseases have in common are highlighted. Finally, emphasis is placed on the gene alterations linked to the deregulation of the PI3K/Akt pathway in DM as a factor of association with $A D$.

\section{Alterations in gene expression in AD}

To better understand the gene alterations involved in $A D$, we must establish key points of the physiopathological process involved in the development of this disease. For this purpose, it is necessary to highlight that there are three important aspects of AD: increased production of the $A \beta$, increased production of hyperphosphorylated Tau protein ( $p$-Tau), and neuroinflammation. From these three factors, the most studied one because of its association with $A D$ is the increased production of $A \beta$, even considering the amyloid hypothesis, which in general terms considers the increased production of this peptide as the starting point for the development of neuroinflammation and the increased production of $\mathrm{p}$-Tau ${ }^{6}$.

Another key point is the influence of two pathways in the processing of amyloid precursor protein (APP): the amyloidogenic pathway through which APP is cleaved by $\beta$ and $\gamma$ secretases, converting it into aggregates where the protease cleavage site is inaccessible within an amyloid structure, preventing normal protease activity with varying but significant degrees of neurotoxicity known as $A \beta$ which have a tendency to aggregate to form amyloid plaques and the non-amyloidogenic pathway through which APP is cleaved by $\alpha$ and $\gamma$ secretly generating soluble forms of APP that is easily degraded and have neuroprotective properties ${ }^{7}$.

Once the key points have been assimilated, we can consider that the genetic alterations have been identified as potential risk factors for the development of $A D$, among them the expression of isoforms of the ApoE gene, mainly those of type 2 and 4 . The importance of these alterations seems to go beyond the increase of the expression of $A \beta$ (which is the mechanism through which they contribute to the development of $A D$ ) since recent studies suggest that the $A p o E$ isoforms interfere in diverse organic functions among which the transcriptional regulation, the neuroinflammation, and the modulation of the activity of the microglia ${ }^{8}$.

Continuing with the alterations associated with the increase in production of $A \beta$ is the genetic variants of both PSEN 1 and 2, which facilitate the processing of APPs by $\gamma$-secretase, which contributes to the formation of $A \beta$ through the amyloidogenic pathway'.

Alterations in the gene encoding APP have also been identified as a potential risk factor for the development of $A D$, mainly early-onset $A D$; the mechanism involved in these APP mutations appears to be the increased affinity for the beta-secretase BACE1, involved in the production of $A \beta$ through the amyloidogenic pathway. Both PSEN 1 and 2 and APP are associated genes in most cases of autosomal dominant $A D$ and to a lesser extent in sporadic early-onset cases ${ }^{10}$.

It has also been identified that over-expression of the neuronal classification receptor SORL1 is associated 
with intracellular accumulation of the amyloid precursor protein from which the peptide is generated $A \beta$. The predisposition to the development of $A D$ due to alterations in the expression of the SORL1 gene depends on the polymorphism present ${ }^{11}$.

Through decision tree analysis, it was identified that alterations of the genes MTHFD1, CASC3, CLIP3, and APP are associated with advanced stages of $A D$, these have an impact on DNA stability, gene translation through alterations in the interaction of the exon with splicing dependent proteins, transport in the Golgi apparatus, and the generation of $A \beta$. In addition, it was observed that overexpression of the MAPT gene is associated with clinical deterioration of the AD patient mainly during the transition from normality to the initial phase of cognitive impairment. It is important to note that this gene is associated with the promotion of inflammation, gliosis, hyperphosphorylation of Tau, and alterations in the neuronal transport of nutrients and organelles $^{12}$.

Analysis of hippocampal neurons from humans with $A D$ has shown decreased expression of RBFOX3, HOMER 1 genes in hippocampal cells from humans with $A D$ accompanied by increased expression of molecules such as GFAP, S100B, AQP4, AIF1, and CD68; the latter indicates glial and microglial activity ${ }^{13}$.

In addition, by determining the most important networks involved in $A D$ in both human subjects and mouse models for $A D(3 x T g-A D-H)$, it was determined that this disease alters the regulatory systems of the immune system at various points. This observation was associated with the persistence of neuroinflammation in $A D$, mainly through the activation of glia by GFAP, VIM, S100B, TGFBR2, TGFBR1, TLN1, LAMP2, CSF1, and $\mathrm{CSF}_{1 \mathrm{R}^{13}}$.

In the mouse model of $A D$, an increase in the expression of long non-coding RNA (LncRNA) of the small nucleolar RNA (snoRNA) type known as e307 (92\% similar to human snoRNA ZL107) and e470 was observed, during the preclinical stage of the development of the alterations, specifically before the formation of the amyloid plaque, and its expression ceases after the appearance of this plaque. According to predictions, both IncARNs appear to induce 2'-O-ribose methylation of ribosomal 18S RNA (rRNA), at positions C798 and U576 as in humans with $A D$, so it is proposed that these IncARNs may serve as early-stage AD markers ${ }^{14}$. The apparent mechanism of association of e307 with the development of $A D$ implies mitochondrial dysfunction since it is located within the intron of a COX7c encoding gene (subunit $7 \mathrm{c}$ of the cytochrome oxidase).
Mitochondrial dysfunction has such an important role in the development of $A D$ that it has been proposed the "hypothesis of the mitochondrial cascade of Alzheimer" that in general terms suggests that the mitochondrial alterations both structural and functional produced by environmental factors and genetic inheritance precede even the phenomena described in the classical theory of the development of $A D$, based on the premise that mitochondrial function affects the expression and processing of APP as well as the accumulation of $A \beta^{15}$.

The most controversial point of this "mitochondrial cascade hypothesis" is that it can only explain the production of $p$-Tau by relying on the "amyloid hypothesis" although independently of this, it justifies the relationship of the mitochondrial dysfunction with the overexpression of Tau through the decrease of the activity of the mitochondrial complex $\mathrm{I}^{16}$.

However, the influence of e307 on mitochondrial dysfunction as part of the mechanisms that favor the development of $A D$ invites us to consider the idea that more IncRNA in the future may be discovered as key pieces of this series of physiopathological events.

The analysis of postmortem brain tissue from subjects with AD in search of NAT-type LncRNA (natural antisense transcription) found 21 positively regulated and 10 negatively regulated, of which EBF3-AS (XLOC_083817) stands out. Moreover, when EBF3-AS was determined in several brain regions, the overexpression was observed in hippocampus, frontal-upper convolution, and entorhinal cortex, areas frequently affected in AD. At the same time, a survey of different types of LncRNA (LncRNA encoded in intergenic regions) was performed, highlighting the significantly increased expression of AD-linc1 which is associated with the regulation of gene expression through epigenetic modifications such as enhancing the regulation of gene expression of neighboring genes and the recruitment of chromatin modifying enzymes to regulate the epigenetic state of specific genomic loci, the major expression of this LncRNA was found in the hippocampus and frontal gyrus, being these also important areas in $A D^{17}$. The role of the IncRNA in $A D$ seems to be even more important from the perspective of the amyloidogenic through since the analysis of cerebral tissue of parietal lobes and cerebellum of humans with $A D$ and in cerebral tissue of mouse Tg19959 model of AD, demonstrated an increase in the expression of BACE1AS type IncRNA, this IncRNA affects the expression of BACE1 by forming a duplex of RNA with the mRNA of BACE1, increasing the stability of this and favoring the expression of this $\beta$-secretase, important in the 
production of $A \beta^{18}$. Hence, non-coding regions seem to have more association with $A D$ than previously thought and fortunately more attention is being paid to those elements that were long dismissed.

\section{Alterations in gene expression in Diabetes Mellitus (DM)}

MicroRNAs (miR) are structures associated with the decrease or cessation of protein transduction through their hybridization to the $3^{\prime}$ region of the coding mRNAs and thus are estimated to regulate almost one-third of the expression of the human genome ${ }^{19}$.

In a group of human subjects with pre-diabetes and DM 2, differential hypermethylation was observed in multiple IncARNs when analyzing DNA from blood samples. Although the specific function of these IncARNs is not yet determined, it is considered that it may interfere with the expression of several genes important for glucose metabolism and other mechanisms affected in $\mathrm{DM}^{20}$.

As demonstrated by the study of miR-29a in mouse model hepatocytes with obesity and DM observing an increase in the expression of this $\mathrm{miR}$ and in hepatocyte culture, it was observed that miR-29a binds to the mRNA encoding p85a of PI3K preventing this phosphorile Akt and thus inactivating the insulin/PI3K/Akt signaling pathway involved in the cellular metabolism of glucose. In turn, inhibition of the insulin/PI3K/Akt pathway is associated with an increase in the expression of the enzyme PEPCK involved in the decrease of gluconeogenesis so that the dysregulation of miR-29a expression impacts on glucose metabolism as part of the pathophysiological process of $\mathrm{DM}^{21}$.

The PI3K/Akt pathway is also influenced by $\mathrm{miR}^{22}$, which targets PTEN by activating Akt, ERK 1 , and ERK 2 by increasing the expression of HIF-1 $\alpha$ and VEGF, in an attempt to compensate for the pseudo-hypoxia conditions present in subjects with DM2, suggesting that miR21 is involved in the development of diabetic retinopathy, one of the most common diabetic complications. Expression of miR21 is increased in subjects with DM2 and is susceptible to inhibition of HIF activity- $1 \alpha^{23}$.

However, miR29a has also been observed in nonobese mouse DM models, so its expression seems to be more associated with DM conditions than with obesity, despite the multiple similarities between both diseases. Along with miR29a in this animal model, an increase in the expression of miR-21, miR-29b miR29c, and miR-34a was also observed. Other miR types whose expression has been observed to be increased in both animal (murine) DM models and cell cultures (pancreatic islet cells) are miR-146 and miR-125a. Regarding miR expression identified in humans, miR-133 and miR-206 were found in myocytes, miR-21 and miR375 in pancreatic cells, and miR-9, miR-29a, miR-30d, miR-34a, miR-124a, miR-146a, and miR-375 in serum from diabetic patients, probably from damaged cells ${ }^{22}$.

The miR375 is important for the survival of pancreatic $\beta$ cells, which corresponds to the observations after the comparative analysis of serological samples from patients with DM2, pre-diabetes, and normal glucose metabolism (NGM), in which an increase in the expression of miR375 was observed, linked to high glucose levels in patients with DM2; however, in preclinical conditions such as pre-diabetes in which glycemia is usually lower, the expression of miR375 did not present significant differences with NGM subjects, which suggests that the mechanism through miR375 of protection of pancreatic cells is activated when glycemia levels fluctuate near the threshold of glycotoxicity since this condition generates considerable damage in the cells of the organism, mainly the pancreatic $\beta$ cells ${ }^{24}$.

It is also interesting to note that increased miR375 expression was positively correlated with increased levels of cholesterol and low-density lipoprotein, suggesting that in conjunction with glucose, these factors may participate in the damage mechanisms that activate pancreatic cells $\beta$ response through increased expression of miR375 as a survival mechanism, which in turn coincides with clinical observations that obesity and dyslipidemias are considered risk factors for the development and progression of $\mathrm{DM} 2^{25}$.

In this same study, miR146 showed a significant positive correlation with increased expression of C-reactive protein, added to the observation of increased expression of this miRNA under conditions of prolonged exposure to hyperglycemia reflected through estimates of $\mathrm{HbA1c}$ with cutoff value greater than $7 \%$, the increased expression of miR146 is due to the conditions of hyperglycemia and that this IncRNA, in turn, participates in the regulation of the expression of pro-inflammatory cytokines $^{26}$ through the reduction of the NF- $\kappa \beta^{27}$. We consider then that both miR375 and miR146 participate in mechanisms of response to the damage caused by the conditions of hyperglycemia and chronic low-grade inflammation associated with DM2.

However, decreased miR expression has also been observed in DM conditions, as in the case of glucose intolerance whose appearance is gradual in the pre-diabetic patient and it has been observed that according 
to the progress of this condition, miR-126 expression gradually decreases, compromising its function as a collaborator in the maintenance of vascular integrity, angiogenesis, and repair of endothelial cells in which it is usually expressed ${ }^{28}$.

This is important if we consider that an important element of the blood-brain barrier is the system of blood vessels that form it and provides evidence for the understanding of vascular damage characteristic of DM and that leads to important complications such as retinopathy, nephropathy, predisposition to cerebrovascular events, and stroke ${ }^{29}$.

Another important element in DM-associated gene alterations is histones. Specifically, histones H3K9ac and $\mathrm{H} 3 \mathrm{~K} 4$ have been observed to respond to increased expression of LncRNA Dnm3os (a human DNM3OS Ortholog) under DM conditions, through acetylation and methylation, respectively so that these histones favor the expression of pro-inflammatory cytosines, which may help us understand some of the multiple pathways that allow the development of the chronic low-grade inflammatory state in the mouse model $\mathrm{DM}^{30}$.

Following the influence of IcnARNs specifically for Dnm3os, another study with DM model mice by induction of RI through hyperlipidic diet showed an increase in Dnm3os expression; moreover, it was observed that under conditions of hyperglycemia and insulin resistance, the promoter region of this IcnRNA interacts with $\mathrm{NF}-\mathrm{Kb}$ promoting the increased expression of this Dnm3os allowing it to alter the expression of genes on various chromosomes (possibly largely through the action of the histones H3K9ac and H3K4 described above) by increasing the expression of pro-inflammatory genes encoded by genes with PU-enriched binding sites. 1, NKx3-2, AP1, STAT, IRF1, REL, and decreasing gene expression with EBF1, REST, and KLF4 type enriched binding sites. Among the genes expressed upward by Dnm3os influence are II6, Tnf, Nos2, and Cd36. In addition, it was observed that the suppression of this IcnRNA decreases the expression of genes such as ITGAX and 116 , being the latter one of the main elements of inflammation ${ }^{30}$.

Together with Dnm3os, LncRNA type E330013P06 (IcnRNA analog of type MIR143HG in humans) has been identified as an important element in the promotion of the low-grade inflammatory state in DM since when analyzing macrophages from DM model mice, an association was observed between increased expression of E330013P06 and macrophage activation, a mechanism that favors the expression of pro-inflammatory cytosines ${ }^{31}$.
NF- $\kappa \mathrm{B}$ is a protein complex that also influences the expression of molecules involved in the inflammatory process through its p65 subunit which is overexpressed in hyperglycemic conditions in a mouse model with hyperglycemia. Among the molecules whose expression is associated with increased NF- $\mathrm{kB}$ p65 are monocyte chemoattractant protein 1 (MCP-1), the pro-inflammatory adhesion molecule ICAM1, IL-6, nitric oxide synthase 2 (NOS 2), and vascular cell adhesion molecule 1 (VCAM-1) important in the chemotaxis of cells of the immune system. Other elements associated with the increase of the expression of the $p 65$ subunit of NF- $\mathrm{KB}$ are the histones $\mathrm{H} 3$ pan-acetylated, acetyl $\mathrm{H} 3 \mathrm{~K} 9$, and acetyl H3K14; however, the increase of these alone does not seem to be responsible for the increase of the molecules MCP-1, ICAM1, IL6, NOS2, and VCAM-1. Since they probably depend on the influence of NF-kB p65 or other mechanisms as in the case of IL6 and NOS2, whose increased expression is also associated with increased Dnm3os ${ }^{32}$.

Regarding gene expression and activity in subjects with DM, it has been estimated that there is an increase in the activity of the PGC-1alpha gene associated with a decrease in the expression of the nuclear respiratory factor (NRF) family, which participates in the regulation of mitochondrial biogenesis, respiratory capacity, and the glucose transport protein GLUT4 in muscle tissue ${ }^{33}$.

However, the range of damage caused by DM conditions to cells is greater since in models of diabetic rats, the expression of anti-apoptotic genes of the $\mathrm{Bcl}-2$ and $\mathrm{Bcl}-\mathrm{xl}$ types is decreased, and the expression of proapoptotic genes of the Bax and Bak types is increased. This indicates the greater tendency of the cells to initiate the apoptotic process in front of the conditions that derive from the alterations of the glucose metabolism. Let us consider as an important group the neurons since the main source of energy for these precisely is glucose ${ }^{34}$.

Another gene involved in DM2 is the proto-oncogene JUN, whose decreased expression in conjunction with increased expression of IL-1beta allows for increased activity of IL 17 and the Toll and NOD type receptors involved in the promotion of the pro-inflammatory state ${ }^{35}$.

The Jun proto-oncogene is then proposed as part of an inflammation regulation system since it favors the transcriptional activation of the tissue inhibitor of metalloproteinase-3 (TIMP-3) which inhibits the TNF-alpha converting enzyme (TACE), thus preventing it from allowing the release of TNF-alpha and thus the activity of this proto-oncogene opposes the inflammatory process; however, this action is interrupted in DM conditions so 
this mechanism could also explain the increase of TNF-alpha in subjects with DM as an important factor in the promotion of the pro-inflammatory state ${ }^{36}$.

\section{Alterations in gene expression in common between DM and AD}

Alterations in glucose metabolism have been an element frequently associated with DM. However, in patients with $A D$, a decrease in the expression of the genes that encode insulin elGF I and II have been observed in brain tissue, as well as a decrease in the expression of the genes that encode insulin elGF-I receptors, which invites us to consider that these alterations in receptor expression have an impact on the normal metabolism of glucose by cells participating in the neurodegenerative process ${ }^{37}$.

According to the evidence obtained from the study of mouse models with a hypercaloric diet and cell cultures of cortical neurons, it seems that the conditions of metabolic stress result in a decrease in the expression of PGC-1, which forms part of the coactive pathway of the peroxisome proliferator-activated gamma receptor (PPARy) and seems to play a role as a negative regulator of the expression of BACE1, a beta-secretase that is significantly involved in the production of $A \beta$ in $A D^{38}$.

At present, through genome-wide association studies (GWAS), it is possible to find nucleotide polymorphisms associated with various diseases and therefore, it is also useful to find the coincidental alterations between two diseases, as in the case of $A D$ and DM.

One of the analyses of several GWAS allowed to find 6 single nucleotide polymorphisms (SNP) rs111789331, rs12721046, rs12721051, rs4420638, rs56131196, and rs66626994 present in both AD and DM2 subjects, these data additionally allow a functional enrichment analysis to determine the metabolic pathways in which these nucleotides intervene, in this case, the lipid metabolism pathway is the most affected by these alterations through alterations in gene expression PPARY, APOC1, and HNF1A ${ }^{39}$.

Other GWAS reported the coincidence of 8 nucleotides for both DM2 and AD; some of these nucleotides were found in the genes TP53INP1, TOMM40, and C8orf38 involved in the development of oxidative stress, an important mechanism that has been pointed out as one of the pathways of association between $A D$ and DM. Another gene involved was FNTAP1 by identifying the nucleotides rs3844143 and rs10898439 near this gene, which expresses farnesyltransferase which has been associated with increased levels of amyloid- $\beta$
$(A \beta)$, neuroinflammation, and impaired cognitive function in mouse models.

Another GWAS analysis allowed the identification of 3 polymorphisms of the RYR3 gene associated with the release of calcium from the endoplasmic reticulum to be internalized in the cell. These polymorphisms seem to link $D M, A D$, and hypertension.

In addition to GWAS studies and functional enrichment analyses for the determination of altered genes and affected pathways, in silico models have proven to be an important tool for acquiring information about genetic alterations in various diseases such as $A D$ and $\mathrm{DM}^{40}$.

One of these predictions in silico that considered the polymorphisms rs1517354 and rs1921915 of one of the nucleotides of the gene that encodes glutaminase as an element present in both $\mathrm{AD}$ and $\mathrm{DM}$ (through previous analysis of databases) alteration that promotes increased expression of the gene that encodes the GLS isoform of glutaminase that is promotes the generation of ammonia and glutamate from glutamine in neurons. Evidence suggesting the neurotoxic role of ammonia as an important element in the neurological impairment present in both $\mathrm{AD}$ and $\mathrm{DM}$; however, this impairment may be associated with the increased glutamate generation evidenced in this study by the excitotoxicity hypothesis.

A process that leads to cell death through mechanisms such as increased internalization of calcium into cells and mitochondrial dysfunction which in the case of neuronal stimulation by glutamate can lead to the induction of necroptosis ${ }^{41}$.

Another experiment that supported the hypothesis of excitotoxicity as a mechanism of neuronal damage in $A D$ and $D M$ consisted of the evaluation of the expression of various LncRNA in the hippocampus of DM model mice, specifically the increased expression of (gi/755552777/ref/XR_875577.1/), which by establishing a dynamic network of IncRNA-mRNA was associated with increased expression of mRNA encoding SLC5A7, a membrane transporter in cholinergic neurons that capture choline for acetylcholine synthesis.

This finding, together with the vulnerability of cholinergic neurons in processes of chronic neurological damage associated with cognitive impairment and the development of dementias such as $A D$, suggests an increased demand on the acetylcholine modulator system in the face of excitotoxicity since excessive activation of NMDA glutamate receptors leads to neurons initiating the process of apoptosis and part of the cholinergic system is likely to try to cope with this demand 
as a neuromodulator, however, eventually, cholinergic neurons will end up "depleting" their capacity and dying, this may explain the susceptibility of cholinergic neurons in models that are excitotoxicity; furthermore, it has been observed that the nicotinic acetylcholine receptor $\alpha 7$ ( $\alpha 7 \mathrm{nAChR})$ has a high affinity for $A \beta$ and is associated with the internalization of this peptide into the cells leading to neurotoxicity by peptide deposit formation ${ }^{42}$.

In addition, in the mouse DM model, a functional enrichment analysis was performed which indicated alterations in the cholinergic pathways, the NF-kB pathway, the TLR4 cascade, and the zinc transporter, which points to other possible pathways through which DM leads to cognitive impairment and through which it may be linked to diseases such as AD.

Regarding to alterations in mRNA expression, we found that in a rat model to which Ri and hyperglycemia were induced by the administration of a high-fat and high-carbohydrate diet, an increase in CDK5 mRNA expression was observed, attributed to an increase in the methylation of the region promoting its gene, as evidenced in the analysis of the brain tissue. It should be noted that CDK5 is an important kinase for Tau phosphorylation which explains why the increase in $\mathrm{p}$-Tau production was also observed during the analysis of the CA1 region of the hippocampus in the $i R$ and hyperglycemic models of this study.

An integrative meta-analysis of transcriptional data sets of subjects with $A D$ and DM type 2 (DM2) of different ages and stages of $A D$ progression was performed. In the data set of adult subjects with DM2 and subjects with advanced $A D$, alterations in the expression of 45 genes were found to be coincident, among which the increased expression of HIGD1A (HIG1 hypoxia inducible domain family, member $1 \mathrm{~A}$ ) and TIA1 (TIA1 cytotoxic granule-associated RNA binding protein) stand out. HIGD1A coincided in subjects with DM2 and $A D$ but not in subjects with $D M 2$ and advanced $A D$ while TIA1 coincided in subjects with DM2 and advanced $A D$ but not in subjects with $D M 2$ and $A D$, so the gene expression that could indicate the coincidence points between DM2 and AD vary according to the state of progression of $A D$ and probably also of $D^{38}$.

Alterations in miR expression in podocyte and endothelial cell cultures exposed to hyperglycemic conditions show a decrease in miR expression of miR93 type, which inhibits the expression of the gene encoding vascular endothelial growth factor (VEFG), decreasing their expression and thus increasing vascular permeability, which can favor the infiltration of pro-inflammatory cytosines and immune cells through vascular structures such as the blood-brain barrier and favor neuroinflammation (Fig. 1) $)^{43}$.

\section{Pathway PI3K/Akt/Gsk3}

Neuroinflammation seems to be an important point in the DM-AD link through several elements among which tumor necrosis factor $\alpha$ (TNF $\alpha$ ) interferes with insulin signaling by preventing phosphorylation of tyrosine residues from the insulin receptor type 1 (IRS 1) substrate and thus inactivating the PI3K/Akt pathway causing iR in the involved tissues, which also decreases the activity of the insulin-degrading enzyme (IDE) by reducing the mRNA encoding this enzyme, which is involved in the clearance $A \beta^{44}$.

The importance of the PI3K/Akt pathway with respect to DM is that most of the metabolic actions of insulin involve PI3K (phosphatidylinositol-3 kinase) and Akt, while its effects on cell differentiation and growth pass through the MAPK (mitogen-activated protein kinase) pathway ${ }^{28}$.

Inhibition of this PI3K/Akt pathway under iR conditions increases the activity of Glycogen Synthase Kinase-3 beta (GSK3 beta) through two mechanisms: preventing phosphorylation of GSK3 beta by Akt and increasing the transcription of mRNA coding for GSK3 beta, which increases its activities, one of which is phosphorylation of Tau generating hyperphosphorylated Tau ( $p-T a u)^{45}$.

A significant decrease in the expression of Akt, a protein important in glucose metabolism, was observed in human subjects with $A D$ and that is subjects with DM2 have great relevance because of its association with the mechanisms of insulin resistance ${ }^{46}$.

In neural cells exposed to insulin increased the expression of mRNA encoding the gene ADAM 10, associated with the generation of alpha-secretase which is involved in the proteolysis of APP at specific sites for the formation of easily degraded substrates. This also implies that the proteolysis of APP does not occur in the T668 site which causes a decrease in the translocation of the ACIDs generated toward the nucleus, important for the decrease of the levels of $A \beta$ since the ACIDs that reach the nucleus induce the transcription of genes involved in the production of APP, GSK3B, BACE 1, and the decrease in the expression of mRNA encoding the IDE, the latter being important for the degradation of the $A \beta$. Simultaneously, exposure to insulin decreased the expression of BACE-1, a beta-secretase involved in the production of $A \beta$. It was further observed that all 


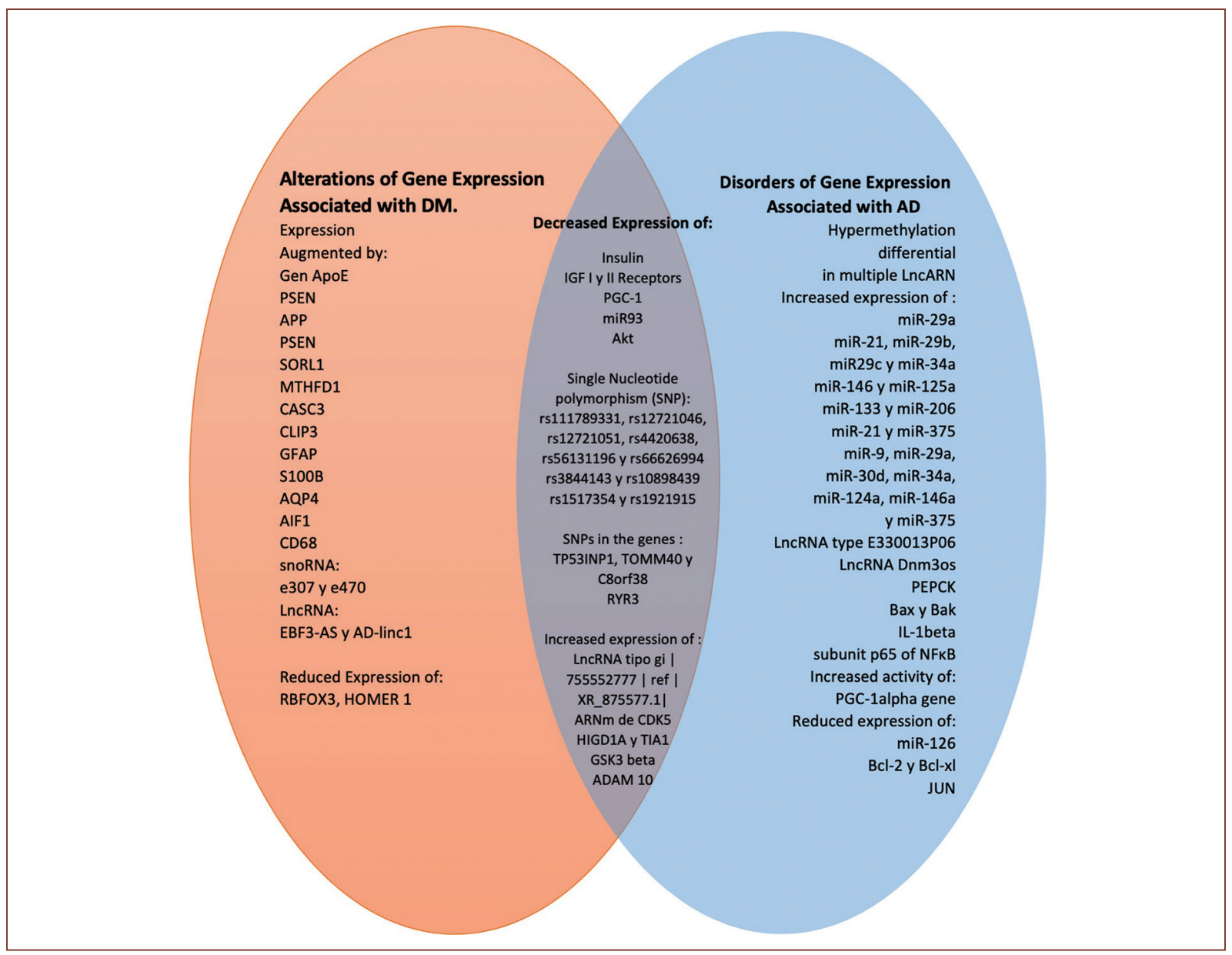

Figure 1. Scheme listing the alterations in gene expression observed in $A D$ models characterized by increased production of $A \beta, p-T a u$, and neuroinflammation (orange oval) and the alterations in gene expression observed in DM conditions such as high glucose concentration and insulin resistance (blue oval) to the center lists alterations in gene expression observed in both $A D$ and $D M$ models that may indicate a link between the two diseases.

these neuroprotective effects are diminished when the $\mathrm{PI} 3 \mathrm{~K} / \mathrm{Akt}$ pathway is interfered with, which is also affected during insulin resistance (Fig. 2).

On the other hand, the characteristic proteinopathy of $A D$ promotes neuroinflammation starting from the fact that $A \beta$ has neurotoxic properties determined by the capacity of aggregation of each subtype being A $\beta 42$ one of the most neurotoxic and more frequently linked to the development of $A D$, together with this peptide, the soluble oligomers of $\beta$-amyloid (A $\beta O s)$ also generated from APP by the amyloidogenic pathway, have been linked to various functional alterations of CNS cells, highlighting in this context their ability to generate a neuroinflammatory response through endothelial and glial activation.

Animal models of $A D$ that is characterized by the absence of amyloid plaques and the presence of both
$\mathrm{A} \beta \mathrm{O}$ s and the diffusible ligands derived from $\beta$-amyloid (ADDL) suggest that these elements influence the neurodegenerative progress from very early stages, even suggesting that both $A \beta O$ s and $A D D L$ have a greater influence on the onset of $A D$ than the insoluble fibrillary oligomers of $A \beta$ deposited in the form of amyloid plaques.

Endothelial activation is characterized by increased expression of immune cell adhesion molecules (ICAM-1 and VCAM-1), vascular endothelial growth factor (VEGF), and prothrombotic factors, matrix metalloproteases (MMP-9 and MMP-2), ROS, AGEs, pro-inflammatory cytosines that activate various pathways including AGE/RAGE/NF- $\mathrm{KB}$ and AGE/RAGE/p21ras leading to vascular inflammation, thrombosis, and increased expression of RAGE. Regarding glial activation, cell lines seem to have an ambivalent role in response to the stimulus of $A \beta$, since when both the 


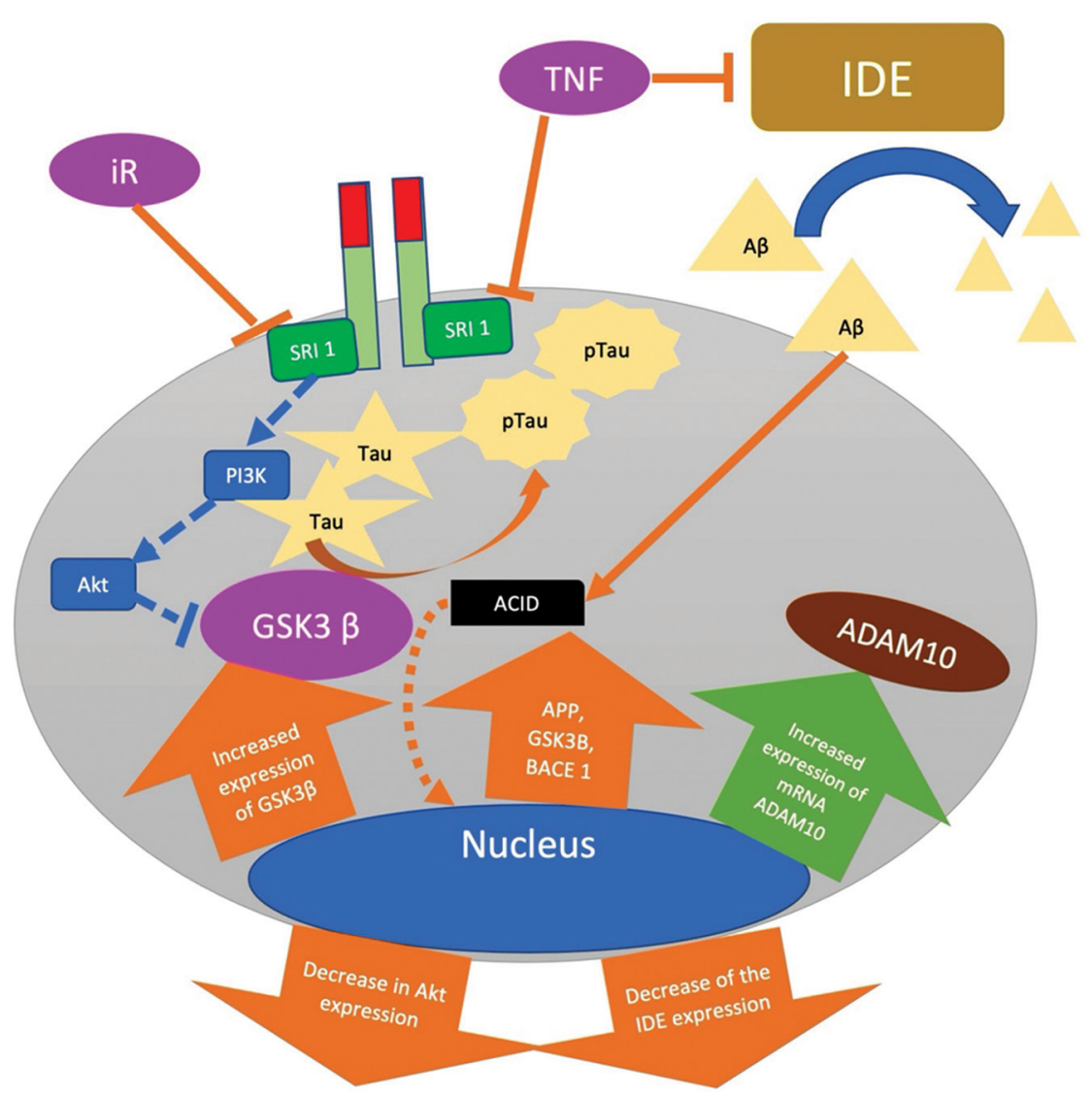

Figure 2. Scheme representing the influence of alterations in gene expression in both $A D$ and $D M$ on the progression of both diseases, thus assuming a link between the two. Insulin resistance (iR) and TNF $\alpha$ (tumor necrosis factor $\alpha$ ) interfere with insulin receptor signaling, preventing activation of the PI3K/Akt (blue dotted line) pathway. In AD models, a decrease in Akt expression has been observed (orange descending arrow); Akt in normal conditions inhibits GSK3 $\beta$; however, in both $D M$ and $A D$, the inhibition of Akt activity translates into an increase in the activity of GSK3 $\beta$, which participates significantly in the process of phosphorylation and hyperphosphorylation of Tau, by increasing its activity it contributes to the increase in the accumulation of $p$-Tau inside the cell. Under IR conditions, an increase in mRNA expression of GSK3 $\beta$ (orange ascending arrow) has been observed. This is an interesting phenomenon considering that both $D M$ and $A D$ have been linked to IR. The correct signaling of insulin seems to impact negatively on the amyloidogenic pathway since it seems to increase the expression of ADAM10 (green upward arrow), a gene encoding an alpha-secretase of APP that interferes with the formation of $A \beta$ (blue arrow) by cleaving APP at specific sites generating soluble and easily degraded elements instead of $A \beta . A \beta$ is also generated under normal conditions and inside the cell are the intracellular domains of APP (ACID) (black box); this can be translocated to the nucleus thanks to GSK3 $\beta$, in conditions such as in DM or AD, in which it is assumed that the activity of GSK3 $\beta$ is increased, translocation of ACID to the nucleus also increases, allowing the nucleus to induce increased expression of APP, GSK3 $\beta$, and BACE 1 (orange upward arrow) important elements of the amyloidogenic pathway, while at the same time inducing decreased expression of IDE (orange downward arrow).

microglia and the astrocytic cells are activated, they express multiple factors, some of which participate in neuroprotection and others in cellular deterioration ${ }^{47}$.

The cells of the microglia generate anti-A $\beta$ antibodies and participate in an important way in the clearing of the plates of $A \beta$. Simultaneously, it releases inflammatory mediators, among which IL-1, IL6, IFN- $\gamma$, TNF- $\alpha$, chemokines, complement components, ROS, and reactive nitrogen species (RNS) and other agents that increase inflammation such as prostaglandin E2, NO, $\mathrm{NF}-\mathrm{KB}, \mathrm{M}-\mathrm{CSF}$, and MCP-1.

As for astrocytes, they present a series of morphological and functional changes in subjects with $A D$ known as reactive astrogliosis, which is characterized 
by hypertrophy and increased production of GFAP, S100B, neurotrophic factors such as thrombospondin-1, activator of tissue plasminogen, transforming growth factor- $\beta$ (TGF $\beta$ ), IL-1, IL-6, ciliary neurotrophic factor and insulin-like growth factor, nerve growth factor (NGF), and an important antioxidant called glutathione (GSH).

However, like microglia, astrocytes simultaneously express cell damage factors such as ROS, advanced glycation end products (AGEs), with the consequent activation of the pathways with the consequent activation of the inflammatory pathways NF-кB and STAT3157, thus enhancing the astrocytic release of cytosines and pro-inflammatory factors such as IL-1, IL-1 $\beta$, IL-6, IL-10, and TNF $\alpha$, interferon- $\gamma(\mathrm{IFN}-\gamma)$, and glutamate, this last factor also linked to the hypothesis of success as a factor of damage and neuronal death ${ }^{48}$.

\section{Discussion}

Interestingly, the main affected pathways identified in several studies were those of lipid metabolism and inflammation.

Regarding the alterations of the lipid metabolism pathway, the PPARY/PGC-1 pathway seems to explain this link through the production of $A \beta$ mainly by decreased expression of the PPAR $Y$ gene. Precisely this gene is interesting because the importance of its alteration has been determined through various methods ranging from database analysis for determining functional enrichment of genes and the determination of affected metabolic pathways to mRNA determinations in tissues from in vivo models and cell culture.

In turn, the endothelial damage characteristic of DM seems to be an important link with $A D$ through alterations of the blood-brain barrier. However, it is important to note that this DM-AD association through damage to the blood-brain barrier is closely related to alterations in the inflammatory process (infiltration of pro-inflammatory cells, chemotaxis, and edema) that translates into neuroinflammation, one of the important elements of $A D$ whose evidence has been studied from a biochemical perspective and which still lacks evidence of what is happening at the level of gene expression.

Precisely the alterations of the inflammation routes are another important element that is present both in $\mathrm{DM}$ and in $\mathrm{AD}$, in this last disease, the astrocytes are an important element since they regulate the neuroinflammatory process in an important way and according to the evidence, the alterations of the gene expression characteristic of $A D$ imply the expression of elements associated with the activation of the astrocytes (gliosis) among which GFAP, S100B, AQP4, AIF1, and CD68 are emphasized.

Another important element of neuroinflammation whose expression is increased in DM and AD is TNF $\alpha$, which is associated with the increase of $A \beta$ and $p$-Tau through interference with SDI expression and inactivation of the PI3K/Akt pathway allowing GSK3b to hyperphosphorylated into Tau. Precisely this ability of TNF $\alpha$ to interfere with the activity of the PI3K/Akt pathway responsible for insulin activity in glucose metabolism allows us to infer that this is a link between alterations in inflammation pathways and insulin resistance evidenced in both DM and AD.

The PI3K/Akt signaling pathway has been associated with insulin activity in glucose metabolism; however, recent evidence has shown that this pathway is involved in the regulation of $p$-Tau production through the expression of GSK3b and $A \beta$ by ACIDs that signal the cell nucleus to increase the expression of APP, BACE1, and ADAM10. Thus, alterations in the gene expression of PI3K/Akt partially explain the phenotypic characteristics of DM and AD. Although the role of the PI3K/Akt pathway is outlined as an important element of the DM$A D$ link, the level of evidence is still not sufficient to ensure that $D M$ has the ability to lead to $A D$ through this pathway.

It should be noted that several studies provide evidence that reinforces the hypothesis of excitotoxicity as a mechanism of neurological impairment involved in both diseases and could even link them, either by increased glutamate or the influence of excitotoxicity on the death of cholinergic neurons in certain regions.

It is important to recognize that the stage of progression of both DM and AD is an important factor in studying the alterations in gene expression that could link both diseases since gene expression varies according to the period of the natural history of each of the diseases.

Although the evidence presented suggests an association between $D M$ gene alterations and $A D$ gene alterations, more evidence is needed to clarify the mechanisms involved in this link.

As mentioned in the case of LncRNA and miR, many of these are just being identified, and the function of each one of them is unknown. Therefore, in the future, alterations in their expression in DM or AD could support or disprove the hypothesis of the DM-AD link.

At the same time, we are in favor of the idea that neuroinflammation and alterations in protein expression 
in $A D$ mainly linked to $A \beta$ have a bidirectional synergic relationship so that the presence of one of these conditions promotes the development and progression of the other condition.

\section{Conclusion}

There are alterations in gene expression that is characteristic of $A D$ and $D M$; however, the number of gene alterations that both diseases have in common is interesting considering that many of these coincide in specific pathways such as lipid metabolism and inflammation, from this perspective, the evidence points to important points that are worth studying further to try to understand the DM-AD link.

It is precisely the alterations in the inflammation pathway that has been associated with alterations in insulin signaling, mainly through the increased expression of TNF $\alpha$ (and other pro-inflammatory cytosines) and the repercussions on the PI3K/Akt pathway.

Furthermore, we can conclude that, although the alterations in gene expression involved in the PI3K/Akt pathway provide evidence of association with important elements of neurological damage through $A \beta$ and $\mathrm{p}$-Tau, the compression of the elements involved in these processes such as miRs, LncRNAs, certain genes, and some nucleotides is not entirely clear, so there is a lack of evidence that allows us to better understand the characteristics of these elements, their role in these mechanisms of disease and the implications of their alterations.

\section{Funding}

KAHC received a doctoral scholarship number 958097 from CONACYT, Mexico.

\section{Conflicts of interest}

The authors declare that there are no conflicts of interest.

\section{Ethical disclosures}

Protection of human and animal subjects. The authors declare that no experiments were performed on humans or animals for this study.

Confidentiality of data. The authors declare that no patient data appear in this article.

Right to privacy and informed consent. The authors declare that no patient data appear in this article.

\section{References}

1. Patterson C. Alzheimer's disease international the global voice on dementia. World Alzheimers Rep. 2018;34:4-7

2. Elahi FM, Miller BL. A clinicopathological approach to the diagnosis of dementia. Nat Rev Neurol. 2017;13:457-76.

3. Spittau B. Aging microglia-phenotypes, functions and implications for age-related neurodegenerative diseases. Front Aging Neurosci. 2017;9:194.

4. Matulewicz N, Karczewska-Kupczewska M. Insulin resistance and chronic inflammation. Postepy Hig Med Dosw (Online). 2016;70:1245-58.

5. Harreiter J, Roden M. Diabetes mellitus-definition, classification, diagnosis, screening and prevention (update 2019). Wien Klin Wochenschr. 2019;131 Suppl 1:6-15.

6. Moore S, Evans LD, Andersson T, Portelius E, Smith J, Dias TB, et al. APP metabolism regulates tau proteostasis in human cerebral cortex neurons. Cell Rep. 2015;11:689-96.

7. Rajmohan R, Reddy PH. Amyloid beta and phosphorylated tau accumulations cause abnormalities at synapses of Alzheimer's disease neurons. J Alzheimers Dis. 2017;57:975-99.

8. Liao $\mathrm{F}$, Yoon $\mathrm{H}, \mathrm{Kim} \mathrm{J}$. Apolipoprotein $\mathrm{E}$ metabolism and functions in brain and its role in Alzheimer's disease. Curr Opin Lipidol. 2017;28:60-7.

9. Braggin JE, Bucks SA, Course MM, Smith CL, Sopher B, Osnis L, et al. Alternative splicing in a presenilin 2 variant associated with Alzheimer disease. Ann Clin Transl Neurol. 2019;6:762-77.

10. Kim JH. Genetics of Alzheimer's disease. Dement Neurocogn Disord. 2018;17:131-6.

11. Gómez-Tortosa E, Ruggiero M, Sainz MJ, Villarejo-Galende A, Prieto-Jurczynska C, Pérez BV, et al. SORL1 variants in familial Alzheimer's disease. J Alzheimers Dis. 2018;61:1275-81.

12. Ferrer I, Andrés-Benito $\mathrm{P}$, Zelaya MV, Aguirre ME, Carmona M, Ausín K et al. Familial globular glial tauopathy linked to MAPT mutations: molecular neuropathology and seeding capacity of a prototypical mixed neuronal and glial tauopathy. Acta Neuropathol. 2020;139:735-71.

13. Castillo E, Leon J, Mazzei G, Abolhassani N, Haruyama N, Saito T, et al. Comparative profiling of cortical gene expression in Alzheimer's disease patients and mouse models demonstrates a link between amyloidosis and neuroinflammation. Sci Rep. 2017;7:17762.

14. Gstir R, Schafferer S, Scheideler M, Misslinger M, Griehl M, Daschil N, et al. Generation of a neuro-specific microarray reveals novel differentially expressed noncoding RNAs in mouse models for neurodegenerative diseases. RNA. 2014;20:1929-43.

15. Swerdlow RH. Mitochondria and mitochondrial cascades in Alzheimer's disease. J Alzheimers Dis. 2018:62:1403-16.

16. Swerdlow RH, Burns JM, Khan SM. The Alzheimer's disease mitochondrial cascade hypothesis: progress and perspectives. Biochim Biophys Acta. 2014;1842:1219-31.

17. Magistri M, Velmeshev D, Makhmutova M, Faghihi MA. Transcriptomics profiling of Alzheimer's disease reveal neurovascular defects, altered amyloid- $\beta$ homeostasis, and deregulated expression of long noncoding RNAs. J Alzheimers Dis. 2015;48:647-65.

18. Faghihi MA, Modarresi F, Khalil AM, Wood DE, Sahagan BG, Morgan TE, et al. Expression of a noncoding RNA is elevated in Alzheimer's disease and drives rapid feed-forward regulation of beta-secretase. Nat Med. 2008; $14: 723-30$

19. Ruiz MA, Chakrabarti S. MicroRNAs: the underlying mediators of pathogenetic processes in vascular complications of diabetes. Can J Diabetes. 2013;37:339-44

20. Pheiffer C, Erasmus RT, Kengne AP, Matsha TE. Differential DNA methylation of microRNAs within promoters, intergenic and intragenic regions of Type 2 diabetic, pre-diabetic and non-diabetic individuals. Clin Biochem. 2016;49:433-8.

21. Pandey AK, Verma G, Vig S, Srivastava S, Srivastava AK, Datta M. miR-29a levels are elevated in the $\mathrm{db} / \mathrm{db}$ mice liver and its overexpression leads to attenuation of insulin action on PEPCK gene expression in HepG2 cells. Mol Cell Endocrinol. 2011;332:125-33.

22. Kong $L$, Zhu J, Han $W$, Jiang $X, X u M$, Zhao $Y$, et al. Significance of serum microRNAs in pre-diabetes and newly diagnosed Type 2 diabetes: a clinical study. Acta Diabetol. 2011;48:61-9.

23. Mazzeo A, Lopatina T, Gai C, Trento M, Porta M, Beltramo E. Functional analysis of miR-21-3p, miR-30b-5p and miR-150-5p shuttled by extracellular vesicles from diabetic subjects reveals their association with diabetic retinopathy. Exp Eye Res. 2019;184:56-63.

24. García-Jacobo RE, Uresti-Rivera EE, Portales-Pérez DP, González-Amaro R Lara-Ramírez EE, Enciso-Moreno JA, et al. Circulating miR-146a, miR-34a and miR-375 in Type 2 diabetes patients, pre-diabetic and normal-glycaemic individuals in relation to $\beta$-cell function, insulin resistance and metabolic parameters. Clin Exp Pharmacol Physiol. 2019;46:1092-100.

25. Zheng Y, Ley SH, Hu FB. Global aetiology and epidemiology of Type 2 diabetes mellitus and its complications. Nat Rev Endocrinol. 2018;14:88-98.

26. Alipour MR, Yousefzade N, Bavil FM, Naderi R, Ghiasi R. Swimming impacts on pancreatic inflammatory cytokines, miR-146a and NF-KB expression levels in Type-2 diabetic rats. Curr Diabetes Rev. 2020;16:889-94. 
27. Gao N, Dong L. MicroRNA-146 regulates the inflammatory cytokines expression in vascular endothelial cells during sepsis. Pharmazie. 2017;72:700-4

28. Hinault $\mathrm{C}$, Dumortier O, van Obberghen E. microRNA and diabetes: tiny things causing huge effects. Med Sci (Paris). 2013;29:785-90.

29. Chow BW, Gu C. The molecular constituents of the blood-brain barrier Trends Neurosci. 2015;38:598-608.

30. Das S, Reddy MA, Senapati P, Stapleton K, Lanting L, Wang M, et al. Diabetes mellitus-induced long noncoding RNA Dnm3os regulates macrophage functions and inflammation via nuclear mechanisms. Arterioscler Thromb Vasc Biol. 2018;38:1806-20.

31. Reddy MA, Chen Z, Park JT, Wang M, Lanting L, Zhang Q, et al. Regulation of inflammatory phenotype in macrophages by a diabetes-induced long noncoding RNA. Diabetes. 2014:63:4249-61.

32. El-Osta A, Brasacchio D, Yao D, Pocai A, Jones PL, Roeder RG, et al. Transient high glucose causes persistent epigenetic changes and altered gene expression during subsequent normoglycemia. J Exp Med. 2008;205:2409-17.

33. Patti ME. Gene expression in the pathophysiology of Type 2 diabetes mellitus. Curr Diab Rep. 2004;4:176-81.

34. Nilsen LH, Rae C, Ittner LM, Götz J, Sonnewald U. Glutamate metabolism is impaired in transgenic mice with tau hyperphosphorylation. J Cereb Blood Flow Metab. 2013;33:684-91.

35. Stylianou E. Epigenetics of chronic inflammatory diseases. J Inflamm Res. 2019;12:1-14.

36. Guinea-Viniegra J, Zenz R, Scheuch H, Hnisz D, Holcmann M, Bakiri L, et al. TNFalpha shedding and epidermal inflammation are controlled by Jun proteins. Genes Dev. 2009:23:2663-74.

37. Steen E, Terry BM, Rivera EJ, Cannon JL, Neely TR, Tavares R, et al. Impaired insulin and insulin-like growth factor expression and signaling mechanisms in Alzheimer's disease-is this Type 3 diabetes. J Alzheimers Dis. 2005;7:63-80.
38. Santiago JA, Bottero V, Potashkin JA. Transcriptomic and network analysis highlight the association of diabetes at different stages of Alzheimer's disease. Front Neurosci. 2019;13:1273.

39. Gao L, Cui Z, Shen L, Ji HF. Shared genetic etiology between Type 2 diabetes and Alzheimer's disease identified by bioinformatics analysis. J Alzheimers Dis. 2016;50:13-7.

40. Jian X, Boerwinkle $\mathrm{E}$, Liu X. In silico prediction of splice-altering single nucleotide variants in the human genome. Nucleic Acids Res. 2014;42:13534-44.

41. Hernández DE, Salvadores NA, Moya-Alvarado G, Catalán RJ, Bronfman FC, Court FA. Axonal degeneration induced by glutamate excitotoxicity is mediated by necroptosis. J Cell Sci. 2018;131:jcs214684.

42. Liu J, Chang L, Song Y, Li H, Wu Y. The role of NMDA receptors in Alzheimer's disease. Front Neurosci. 2019;13:43.

43. Long J, Wang Y, Wang W, Chang BH, Danesh FR. Identification of microRNA-93 as a novel regulator of vascular endothelial growth factor in hyperglycemic conditions. J Biol Chem. 2010;285:23457-65.

44. Baglietto-Vargas D, Shi J, Yaeger DM, Ager R, LaFerla FM. Diabetes and Alzheimer's disease crosstalk. Neurosci Biobehav Rev. 2016;64:272-87.

45. Zhang Y, Huang NQ, Yan F, Jin H, Zhou SY, Shi JS, et al. Diabetes mellitus and Alzheimer's disease: GSK-3 $\beta$ as a potential link. Behav Brain Res. 2018;339:57-65.

46. Liu SY, Zhao HD, Wang JL, Huang T, Tian HW, Yao LF, et al. Association between polymorphisms of the AKT1 gene promoter and risk of the Alzheimer's disease in a Chinese Han population with Type 2 diabetes. CNS Neurosci Ther. 2015;21:619-25.

47. Mason JL, Suzuki K, Chaplin DD, Matsushima GK. Interleukin-1beta promotes repair of the CNS. J Neurosci. 2001;21:7046-52.

48. Hwang IK, Choi JH, Nam SM, Park OK, Yoo DY, Kim W, et al. Activation of microglia and induction of pro-inflammatory cytokines in the hippocampus of Type 2 diabetic rats. Neurol Res. 2014;36:824-32. 